\title{
EL DIRECCIONAMIENTO ORGANIZACIONAL FUNDAMENTADO EN LOS SISTEMAS DE GESTIÓN INTEGRAL: UNA RELACIÓN ESTRATÉGICA PARA LA MEJORA
}

\section{ORGANIZATIONAL MANAGEMENT BASED ON INTEGRATED MANAGEMENT SYSTEMS: A STRATEGIC RELATIONSHIP FOR IMPROVEMENT}

\author{
MSc.Yurley Contanza Medina Cárdenas, MSc Yesenia Areniz Arévalob, MSc. Edgar Antonio \\ Sánchez Ortiz ${ }^{\mathrm{c}}$ \\ ${ }^{a}$ Universidad Francisco de Paula Santander Ocaña, GRIITEM \\ Ocaña, Colombia, ycmedinac@ufpso.edu.co \\ ${ }^{\mathrm{b}}$ Universidad Francisco de Paula Santander Ocaña, GRIITEM \\ Ocaña, Colombia, yareniza@ufpso.edu.co \\ ${ }^{c}$ Universidad Francisco de Paula Santander Ocaña, INGAP \\ Ocaña, Colombia, direccion@ufpso.edu.co
}

\begin{abstract}
Resumen: El ideal de progreso definido en la visión organizacional, se logra cuando hay bases estructurales establecidas; como el análisis del contexto, la definición de objetivos, el establecimiento de metas, políticas y estrategias que direccionan la capacidad para adaptarse y responder a los cambios del entorno.

En el caso de las instituciones de educación superior IES, la calidad es un imperativo desde todos sus ángulos, se espera una gestión administrativa eficiente y una formación académica pertinente de altos estándares y competencias.

Los resultados parten de una investigación concluyente - descriptiva, sobre el nivel de apropiación del mejoramiento, visto como la capacidad de autogestión organizacional que estudiantes, docentes y personal administrativo conceptuaron sobre el nivel de madurez en cuanto a los aspectos de la calidad institucional a partir del contexto interno y la política integral de una Universidad objeto de estudio.
\end{abstract}

Palabras clave: Autogestión, Calidad, Educación Superior, Estrategias, Política Institucional. 


\begin{abstract}
The ideal of progress defined in the organizational vision, is achieved when there are established structural basis; as context analysis, defining objectives, establishing goals, policies and strategies, that orientate the ability to adapt and respond to the changing environment.

In the case of institutions of higher education IES, quality is an imperative from all angles, it expected an efficient administration and a relevant academic training of high standards and competencies.

The results are based on a conclusive - descriptive research on the level of appropriation of betterment, seen as the ability of self management organizational that students, students, teachers and administrative personnel conceptualized on the level of maturity regarding aspects of institutional quality from the internal context and integrated policy of a university under study.
\end{abstract}

Keywords: Self-Management, Quality, Higher Education, Strategies, Institutional Policy.

\section{INTRODUCCIÓN}

La relación estratégica analizada en esta investigación, corresponde a una mirada prospectiva cuyo objetivo consistió en identificar oportunidades de desarrollo sostenible a partir del nivel de madurez en cuanto a la capacidad de gestión institucional para formular, implementar y mantener estrategias de mejora continua y excelencia académico - administrativa.

Teniendo en cuenta resultados arrojados de un proceso de evaluación integral que consideró el contexto institucional interno (elementos claves de la gestión y principios de la calidad) y los ejes de la política de gestión de la Universidad, objeto de estudio; se conceptúa sobre los aspectos generales que deben ser tenidos en cuenta para dar cumplimiento a los objetivos alineados con la estrategia de la institución.

En cuanto al contexto interno se muestran los aspectos evaluados y el grado de madurez considerado por los 3 actores consultados (estudiantes, docentes y personal administrativo), destacando las debilidades y fortalezas de la Universidad.

En cuanto a la política integral en sus tres ejes (ambiental, calidad y salud ocupacional), se evaluó el nivel de apropiación que los actores tienen al respecto, considerando la importancia de los sistemas de gestión y su alineación con la estrategia institucional.

Esto fue importante para constatar lo mencionado por (Vargas et al, 2005: 3), al analizar la trasformación de los procesos en la formulación de estrategias con relación a la evolución de la organización, siendo necesario que la alta dirección responda a la creciente demanda del entorno mediante un proceso continuo de innovación en todas las actividades de la empresa.

Así mismo, el determinar el grado de madurez y el nivel de apropiación a partir de 
lo que sus principales actores perciben de la gestión institucional, es fundamental para que sean ellos mismos quienes determinen cómo su rol encaja en el direccionamiento estratégico y de esta manera contribuyan al logro de los objetivos, (Crosby, 1997).

El enfoque bajo el cual se realizaron los análisis, tuvo en cuenta que la institución debe responder por aspectos relacionados con su desarrollo sostenible, pertinente y eficaz a todas sus partes interesadas, en tal sentido los ejercicios realizados desde la perspectiva de los diferentes actores, permitió identificar las brechas entre lo que la Universidad ha logrado y lo que quiere lograr en coherencia con su direccionamiento estratégico.

Esto es importante según (Jarillo, 1992), porque con base en estos elementos la organización debe tomar la decisión estratégica del tipo de ventaja competitiva que quiere obtener.

Dentro del estudio se tuvieron en cuenta tres técnicas, la encuesta, el autodiagnóstico y la autoevaluación. Estas dos últimas fueron necesarias para comprobar el grado de percepción cualitativo y cuantitativo de los tres estamentos frente a lo que se considera bien hecho y además va acompañado de conceptos particulares sobre lo que debería ser, lo que hace falta y lo que puede ser mejor, (Cabero et al, 2002).

En tal sentido, el grado de objetividad en el análisis fue dado en la comparación y contraste de la valoración y la opinión de los actores en sus diferentes roles, frente a los criterios (situación ideal) contra los cuales se les permitió comparar (Núñez et al, 2012).

\section{METODOLOGÍA}

\subsection{Determinación del contexto institucional.}

Al determinar el contexto institucional y proceder a su evaluación, fue necesario considerar las variables: principios de la gestión de calidad y elementos claves de la gestión de acuerdo a las ISO 9004.

Para el caso de la política integral, en el contexto institucional se consideraron los ejes de la gestión de calidad, la gestión del medio ambiente y la gestión de la seguridad y salud en el trabajo con sus respectivos objetivos.

\subsection{Determinación de la población.}

Para el nivel de apropiación de la política integral, el Universo estuvo conformado por la población activa de la institución objeto de estudio; se estableció la cantidad por estamento y el índice de participación de acuerdo a las encuestas realizadas; distribuidas como se muestra en la tabla 1.

Tabla 1. Descripción de la población encuestada

\begin{tabular}{|l|c|c|}
\hline \multicolumn{1}{|c|}{ Estamento } & $\begin{array}{c}\text { Encuestas } \\
\text { registradas }\end{array}$ & $\begin{array}{c}\text { Porcentaje de } \\
\text { participación }\end{array}$ \\
\hline Estudiante & 2496 & $85,92 \%$ \\
\hline Docente & 264 & $71,73 \%$ \\
\hline Administrativo & 145 & $38,77 \%$ \\
\hline
\end{tabular}

Para el desarrollo del autodiagnóstico de la gestión dentro del contexto interno, la participación en 8 mesas de trabajo interdisciplinares estuvo compuesta, como se muestra en la tabla 2. 
Tabla 2. Descripción de la población participante en la evaluación

del contexto

\begin{tabular}{|l|c|}
\hline \multicolumn{1}{|c|}{ Estamento } & \multicolumn{1}{c|}{$\begin{array}{c}\text { Porcentaje de } \\
\text { participación }\end{array}$} \\
\hline Estudiante & $43,21 \%$ \\
\hline Docente & $63,14 \%$ \\
\hline Administrativo & $92 \%$ \\
\hline
\end{tabular}

Fuente: Elaboración propia

\subsection{Diseño del instrumento de recolección de datos.}

El instrumento utilizado para el autodiagnóstico de la gestión (contexto interno), correspondió a una matriz distribuida entre los principios de la gestión de calidad (8) según ISO 9001:2008 y los elementos claves de la gestión (6) según ISO 9004. Para cada aspecto a evaluar se estableció una escala de Likert con puntuaciones de 1 a 5 en la cual los actores del proceso consideraban el nivel de madurez de la Universidad frente a las preguntas formuladas.

Tabla 3. Autodiagnóstico elementos claves de la gestión

\begin{tabular}{|c|l|l|l|}
\hline \multicolumn{2}{|c|}{ Elemento clave } & Meta & $\begin{array}{l}\text { Nivel de } \\
\text { madurez }\end{array}$ \\
\hline \multirow{3}{*}{ Gestión } & $\begin{array}{l}\text { ¿Cuál es el centro } \\
\text { de interés de la } \\
\text { dirección? }\end{array}$ & & \\
\cline { 2 - 3 } & $\begin{array}{l}\text { ¿Cuál es el } \\
\text { enfoque del } \\
\text { liderazgo? }\end{array}$ & & \\
\hline
\end{tabular}

Fuente: Elaboración propia

Dentro de los elementos claves se consideraron: gestión, estrategia y política, recursos, procesos, seguimiento y medición, mejora, innovación y aprendizaje. La meta o el ideal fue la calificación máxima a obtener de 5 .

Para determinar el nivel de apropiación, se utilizó un formato de encuestas por bloques en la que se distribuyó cada factor evaluado y se indagó a través de afirmaciones para ser calificados según el grado de aceptación, en escala de 1 a 5; la encuesta se realizó en línea, de forma anónima clasificando el tipo de comunidad que representaba y ampliando su percepción con comentarios personales sobre el factor de cada eje en la política integral.

\subsection{Recopilación y análisis de los datos.}

Los resultados de la matriz de valoración del contexto interno (principios y elementos claves), fueron recopilados y consolidados por cada estamento participante para determinar un promedio con la relación entre el ideal (5) y la puntuación asignada.

Los resultados de las encuestas fueron tomados del aplicativo de encuestas del Sistema de Información para la Gestión y Desempeño Institucional SIGDI, http://autoevaluacion.ufpso.edu.co/encuestas /. En el análisis se tuvo en cuenta el grado de apropiación otorgado a cada elemento del direccionamiento, para aquellos datos que fueron calificados como "mayormente de acuerdo" (4) y "totalmente de acuerdo" (5). Se tomaron los comentarios individuales que merecieron las diferentes apreciaciones de los grupos de interés para profundizar en las razones sobre las cuales se basó su concepto.

\section{RESULTADOS}

\subsection{Autodiagnóstico principios de la gestión de calidad}

Evaluados los 8 principios dispuestos en la ISO 9001:2008, se obtuvieron los resultados mostrados en la figura 1 . 


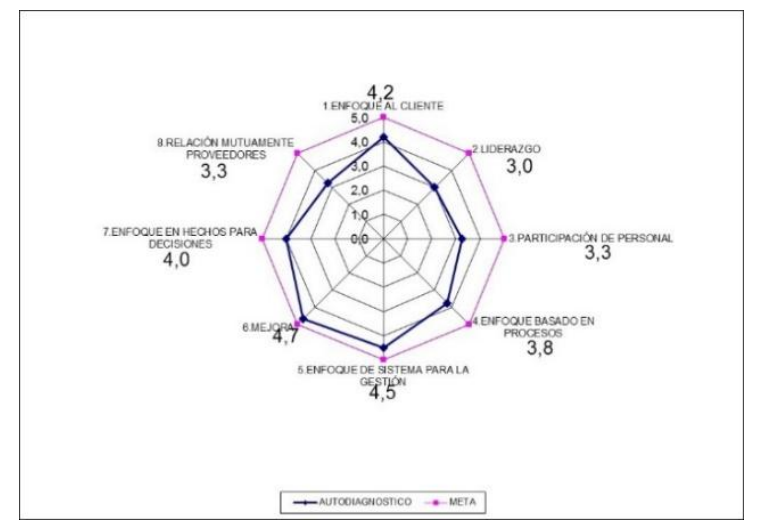

Figura 1. Puntuación por principio de gestión

Fuente: Elaboración propia

Los tres estamentos en promedio, consideraron que la Universidad se encuentra muy cercana al ideal en los conceptos de mejora, enfoque del sistema para la gestión y enfoque al cliente.

Además afirmaron, que estos elementos se encuentran inmersos en la misión institucional y que esto ha conllevado a que las capacidades organizacionales sean asumidas como propias y se contribuya a ellas de forma proactiva; con respecto a esto (Rodríguez et al, 2013), han expuesto que la relación entre lo misional y las capacidades organizacionales son elementos fundamentales en el diseño de la estrategia y su implementación, por lo tanto impactan definitivamente en la calidad de las universidades.

Finalmente, el principio de mejora que se encuentra en un $93 \%$ de madurez, debe ser fortalecido desde la planeación individual de los funcionarios como un objetivo específico de sus funciones.

El promedio general de madurez de la Universidad con respecto a los principios de la gestión de calidad es del 77\%, siendo el liderazgo el de menor puntuación, observado en el estamento administrativo. Esto se evidencia en la debilidad observada desde el seguimiento que debe llevarse a cabo por parte de la alta dirección sobre lo que se comunica y cómo se comunica para asegurar un entendimiento general de los propósitos institucionales.

\subsection{Autoevaluación de los elementos claves de la gestión}

Se evaluaron los 6 elementos claves sugeridos en la ISO 9004, encontrando en promedio los siguientes resultados.

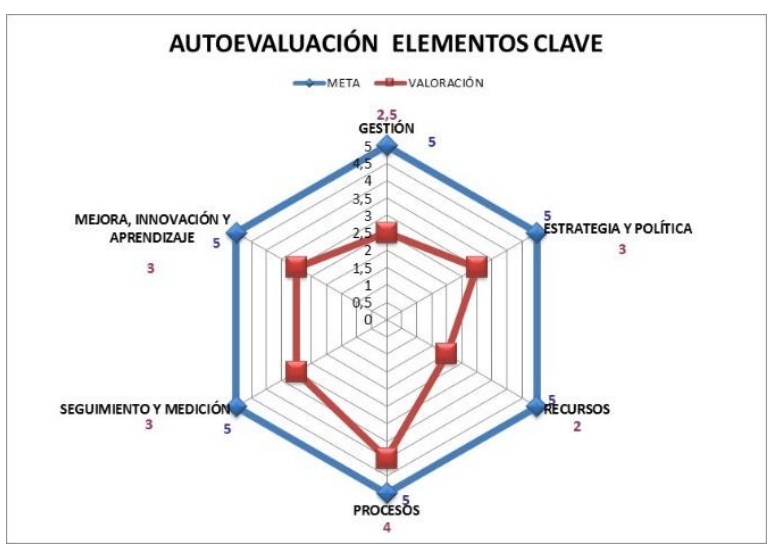

Figura 2. Nivel de madurez de los elementos de la gestión

Fuente: Elaboración propia

Los 3 estamentos evaluaron los elementos considerando que la Universidad aún debe mejorar en la forma como se planifican los recursos y les hace seguimiento.

Estas actividades son de gran importancia en la formulación estratégica pues afecta la proyección a largo plazo como lo mencionan (Acevedo et al, 2010) sobre la necesidad de adecuar estrategias factibles y deseables tomando en consideración los recursos y la forma como se planean y asignan.

Por otra parte (Medina et al, 2015) afirman que esto es coherente con una visión que sea percibida como pertinente y armonizada con 
las capacidades institucionales; nivel que se logra mediante el aprendizaje y la madurez adquiridas en un periodo de tiempo.

El mayor nivel de madurez se identifica en los procesos y esto se evidencia en los sistemas de gestión implementados que han permitido optimizar y organizar métodos de trabajo, además de construir una cultura de planeación, evaluación, seguimiento y mejoramiento continuo que se ha visto reflejado en significativos logros y resultados alcanzados, esta percepción es predominante en estudiantes y docentes participantes de la autoevaluación.

Por su parte los administrativos argumentan que ha sido la tecnología que da soporte a los métodos de trabajo más eficaces y eficientes, la responsable de que se perciba un mejoramiento continuo y un enfoque al cliente más aterrizado. Con respecto a este argumento (Aguilera et al, 2009) confirman que la tecnología es una herramienta fundamental del éxito en el direccionamiento estratégico, dadas las relaciones de este con el cliente y el entorno, entendidas como un todo.

\subsection{Nivel de apropiación de la política integral.}

La política es entendida como los compromisos cuantificables con la responsabilidad social y está asociada al desarrollo sostenible bajo principios de calidad, impacto social y cuidado del medio ambiente. Según (Rangel et al, 2012) "La Universidad tiene la capacidad de difundir y poner en práctica una serie de principios y valores por medio de sus procesos de gestión y son consideradas variables estratégicas de su desarrollo". En tal sentido esos compromisos serán asumidos y exitosos en la medida en que las personas los asuman como parte de su conducta, sus valores y principios.

3.3.1 Eje de calidad. El eje de calidad es el factor institucional de mayor apropiación cercano al $76 \%$ dentro de los tres estamentos universitarios. Se expresa coherencia, pertinencia, claridad y articulación con la naturaleza y objetivos de la Institución; se percibe difundido y permeado en los ámbitos académicos y administrativos.

"La investigación como eje de desarrollo institucional", "la idoneidad del recurso humano y garantizar la satisfacción de los requerimientos de nuestros clientes" son los compromisos mejor adoptados y de los cuales afirman mayor avance los últimos años, coincidiendo en que son estos compromisos los que permitirán mantener el mejoramiento continuo de la gestión y el logro de la excelencia.

Los estamentos se sienten partícipes de los procesos de la calidad principalmente por la autoevaluación institucional que reconocen como una estrategia efectiva de evaluación; sin embargo, consideran que los mecanismos de comunicación no han logrado ser eficaces como se expresa en la política ya que requieren mayor organización e implementación para lograr ser transversales y transmitir información oportuna; otro aspecto por mejorar es la evaluación y selección de personal para dar garantía de la idoneidad que se requiere.

Los estudiantes y docentes argumentan percibir esfuerzo por la calidad en términos de gestión, pero poca divulgación acerca del compromiso institucional en términos pedagógicos, tecnológicos, bibliográficos y curriculares. Ver Figura No 3. 


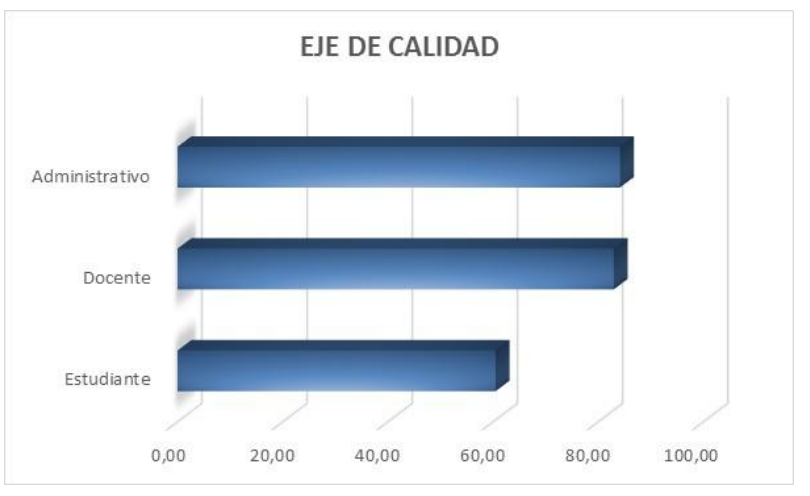

Figura 3. Nivel de apropiación del eje de calidad.

Fuente: Elaboración propia

3.3.2 Eje de seguridad y salud en el trabajo. Este eje tiene una apropiación general del $73.58 \%$, resaltando que sus compromisos son adecuados y claros acerca de la función y la responsabilidad que la Institución tiene frente a la seguridad, la salud y el bienestar de su comunidad universitaria.

Los encuestados tienen una percepción positiva y clara acerca del bienestar y la seguridad del talento humano en su ambiente laboral y para garantizar la protección y conservación de la salud, principalmente los administrativos han visto actividades significativas orientadas a fortalecer este aspecto. Sin embargo, argumentan la necesidad de mejorar el tema de prevención de accidentes de trabajo y el seguimiento a las enfermedades laborales y comunes del personal.

Los tres estamentos coinciden en un $50.43 \%$ en que falta mayor socialización y trabajo con la comunidad para garantizar una óptima y oportuna implementación de los programas de salud ocupacional, argumentan falta de señalización en el campus y en las oficinas, así como puestos de trabajo que no están acondicionados suficientemente para el desarrollo de las jornadas laborales. Manifiestan la necesidad de socializar resultados sobre los objetivos trazados y la evolución que se ha tenido al respecto del cuidado y bienestar de la comunidad. Ver Figura No 4.

EJE DE SEGURIDAD Y SALUD

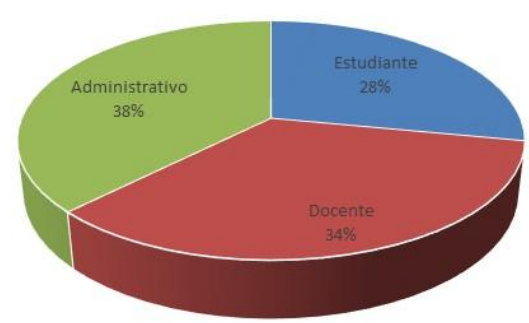

Figura 4. Nivel de apropiación del eje de SYSO.

Fuente: Elaboración propia

3.3.3 Eje ambiental. El eje ambiental arrojó un $72.86 \%$, sin embargo es el factor de menor nivel de apropiación entre la comunidad universitaria. Como aspectos positivos se destaca el reconocimiento que los estamentos tienen acerca del compromiso institucional con la protección y conservación del ambiente a través de las actividades de mantenimiento físico.

En general coinciden en que se percibe un ambiente saludable, bien tratado $\mathrm{y}$ responsable ambientalmente $y$ que es pertinente la estrategia de incorporar la ética ambiental en todas las actividades docentes, investigativas y de servicios; sin embargo, argumentan desconocer el desarrollo y los resultados de los programas ambientales, no es clara la eficiencia alcanzada en el uso de los recursos naturales, se percibe una desarticulación entre el programa de ingeniería ambiental, sus estudiantes y el sistema de gestión ambiental siendo contradictorio para el impacto del programa en la sociedad. 
El $53.89 \%$ está pocas veces de acuerdo en que se esté trabajando en la toma de conciencia, pues argumentan que sin educación ambiental difícilmente se logrará permear la responsabilidad de forma individual especialmente con los estudiantes $\mathrm{y}$ administrativos.

El personal administrativo expresa claramente tener cuidado de sus actividades laborales para minimizar el impacto que estas puedan causar, aunque mencionan que han sido limitadas las campañas y el fomento pues sólo se ha involucrado el tema del uso del papel sin mostrar seguimiento y resultados. Ver Figura No 5.

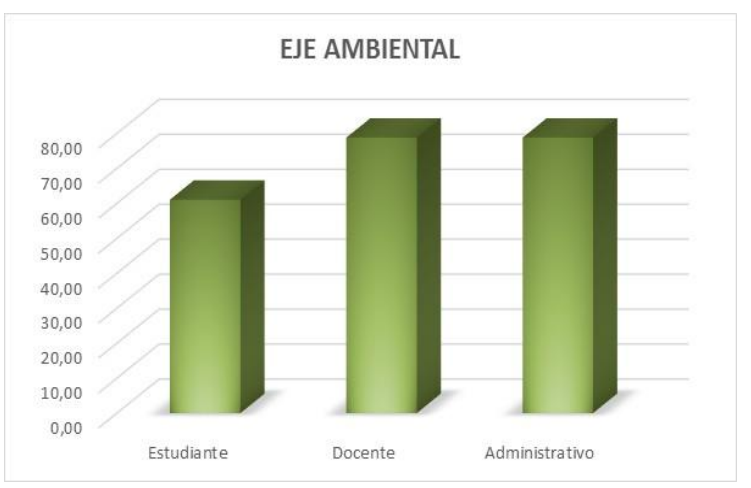

Figura 5. Nivel de apropiación del eje del SIGA.

Fuente: Elaboración propia

(Rodríguez, 2009), en su estudio sobre la influencia del entorno en el desarrollo del direccionamiento estratégico, hace énfasis en la importancia de que las empresas analicen factores de tipo social, tecnológico, cultural, económico y ecológico para garantizar una gestión estratégica, pertinente y coherente.

Esto evidencia que así como la calidad es un aspecto conocido, difundido y apropiado, la toma de conciencia frente al medio ambiente y el bienestar de las personas influye directamente en cómo se percibe la calidad, de forma integral orientada a la gestión académico - administrativa y también a las condiciones del entorno que influyen en los recursos naturales que se utilizan y en las personas.

Una política que refleje los compromisos frente a la naturaleza propia de una Universidad y que tenga en cuenta además, que necesita cuidar los recursos humanos y ambientales con que cuenta; es una política con la cual habrá compromiso, afinidad y respeto, como lo mencionaron los estamentos.

\section{CONCLUSIONES.}

Esta investigación permitió, determinar la pertinencia de tres momentos y métodos de evaluación institucional e identificar la viabilidad de estas herramientas para determinar las fortalezas y debilidades de la Universidad en sus mecanismos de gestión, así como la eficacia de estos para proveer información veraz de la mejora y el nivel de cumplimiento de sus compromisos declarados.

La política integral articula los objetivos estratégicos, aquellos que definen la forma como la Universidad cumplirá y avanzará hacia sus metas; los cuales son percibidos como pertinentes, adecuados y de eficaz contribución al avance de la institución durante los últimos años. Sin embargo, se evidenció la falta de seguimiento como un aspecto no maduro, lo que puede constituir un riesgo para la ejecución de los objetivos.

Los actores del proceso se identifican con el direccionamiento estratégico, entre sus objetivos y los institucionales existe interdependencia y es evidente la comprensión del concepto de calidad académico - administrativa, asociado a una 
responsabilidad individual de oportunidad de servicio.

\section{FINANCIACIÓN}

La División de Investigación y Extensión (DIE) de la Universidad Francisco de Paula Santander Ocaña (UFPSO), apoya la ejecución y desarrollo de proyectos de investigación a los diferentes estamentos. El presente artículo muestra los resultados de una fase del proyecto inscrito, avalado $\mathrm{y}$ financiado en dicha dependencia llamado "Estrategias para el mejoramiento de la gestión académico - administrativa en la educación superior", propuesto por el Grupo de Investigación en Ingenierías Aplicadas (INGAP).

\section{BIBLIOGRAFÍA}

Acevedo, A. et al (2010). "Modelo de análisis y formulación estratégica. Empleando herramientas matriciales". Industrial Data, vol. 13, núm.1, 9-17.

Aguilera, A. et al (2009). "Direccionamiento estratégico apoyado en las TIC". Estudios Gerenciales, vol. 25, núm. 111, 127-143.

Cabero, J. et al (2002). "La evaluación, materiales formativos multimedia en la red. Guía práctica para su diseño". Redalyc, 217.

Contreras, E. (2013). "El concepto de estrategia como fundamento de la planeación estratégica". Pensamiento y Gestión, núm. 35, 152-181.

Crosby, P. (1997). "Gestión, Calidad y Competitividad". Aravaca (Madrid): McGraw-Hill.
Dess, G. et al (2011). "Administración Estratégica". México D.F: Mc-Graw Hill.

González, J. et al (2011). "Análisis estructural Integrativo de Organizaciones Universitarias". Riev S.C. Vol. I.

Hernández, J. R. (2012). "Administración estratégica de la visión a la ejecución". Del Valle, México: Alfa omega.

Ivancevich, J. et al (1997). "Gestión, Calidad y Competitividad". Aravaca (Madrid): McGraw-Hill.

Jarillo, J. C.. (1992). "Dirección Estratégica". Aravaca (Madrid): McGraw-Hill.

López, José et al (2012). "Ambiguedad organizacional en la planeación estratégica". FACES Journal, vol. 11, núm. 2, 44-67.

Núñez, F. et al (2012). "Importancia de la Evaluación y Autoevaluación en el rendimiento académico". Revista del Instituto de Estudios en Educación Universidad del Norte, 96-104.< http://www.redalyc.org/pdf/853/85323 935009.pdf> [Consulta: nov. 2015].

Rangel, O. et al (2012). "Ética como enfoque de la responsabilidad social: direccionamiento estratégico en universidades públicas". Omnia, vol.18, núm. 2, 95-108.

Rodríguez, A. (2009). "Influencia del entorno en el desarrollo del direccionamiento estratégico". Cuadernos de Administración, núm. $42,1-18$.

Rodríguez, E. et al (2013). "Dirección Estratégica y Calidad de las 
Universidades: Un estudio exploratorio desde Chile". Interciencia, vol. 38, núm. 1, 35-41.

Valencia, M. (2013). "Generación y transferencia de conocimiento". Ingeniería industrial, vol. XXXIV, núm. 2, 178-187.

Vargas, J. et al (2005). "Los procesos de transformación estratégica en relación con la evolución de las organizaciones". Estudios Gerenciales, núm. 94, 1-5.

Zapata, R. (2008). "Lineamientos metodológicos para la formulación del plan de desarrollo estratégico y prospectivo". Ciencias Estratégicas, vol. 16, núm. 19, 147-173. 